\title{
Positive cross-correlations in a three-terminal quantum dot with ferromagnetic contacts
}

\author{
A. Cottet, W. Belzig, and C. Bruder \\ Department of Physics and Astronomy, University of Basel, Klingelbergstrasse 82, 4056 Basel, Switzerland
}

(Dated: October 31, 2018)

\begin{abstract}
We study current fluctuations in an interacting three-terminal quantum dot with ferromagnetic leads. For appropriately polarized contacts, the transport through the dot is governed by a novel dynamical spin blockade, i.e., a spin-dependent bunching of tunneling events not present in the paramagnetic case. This leads for instance to positive zero-frequency cross-correlations of the currents in the output leads even in the absence of spin accumulation on the dot. We include the influence of spin-flip scattering and identify favorable conditions for the experimental observation of this effect with respect to polarization of the contacts and tunneling rates.
\end{abstract}

PACS numbers: 73.23.-b,72.70.+m,72.25.Rb

Quantum fluctuations of current in mesoscopic devices have attracted considerable attention in the last years (for reviews, see Refs. [1, 2]). It has been shown that the statistics of non-interacting fermions leads to a suppression of noise below the classical Poisson value [3, [4, [5] and to negative cross-correlations in multi-terminal structures [6]. This was recently confirmed experimentally in a Hanbury Brown-Twiss setup [7]. The question of the sign of cross-correlations has triggered a lot of activity 8], and different mechanisms to obtain positive crosscorrelations in electronic systems have been proposed. Employing a superconductor as a source, positive crosscorrelations have been predicted for several setups [9]. This is because a superconducting source injects highly correlated electron pairs. Screening currents due to longrange Coulomb interactions lead to positive correlations in the finite-frequency voltage noise measured at two capacitors coupled to a coherent conductor [8, 10]. Lastly, positive cross-correlations can occur due to the correlated injection of electrons by a voltage probe [12], or due to correlated excitations in a Luttinger liquid [13].

Below we will be interested in noise correlations in a quantum dot. This problem was addressed theoretically in the sequential-tunneling limit 14 and in the cotunneling regime 15. Noise measurements 16 were in agreement with the Coulomb-blockade picture 14. Crosscorrelations between particle currents in a paramagnetic multi-terminal quantum dot were studied in Ref. [17], and they were found to be negative. The noise of a twoterminal quantum dot with ferromagnetic contacts was studied in the sequential tunneling limit 18, 19], and, interestingly, a super-Poissonian Fano factor was found.

In this Letter, we consider an interacting threeterminal quantum dot with ferromagnetic leads. The dot is operated as a beam splitter: one contact acts as source and the other two as drains. Our main finding is that sufficiently polarized contacts can lead to a dynamical spin blockade on the dot, i.e., a spin-dependent bunching of tunneling events not present in the paramagnetic case. A striking consequence of this spin blockade is the

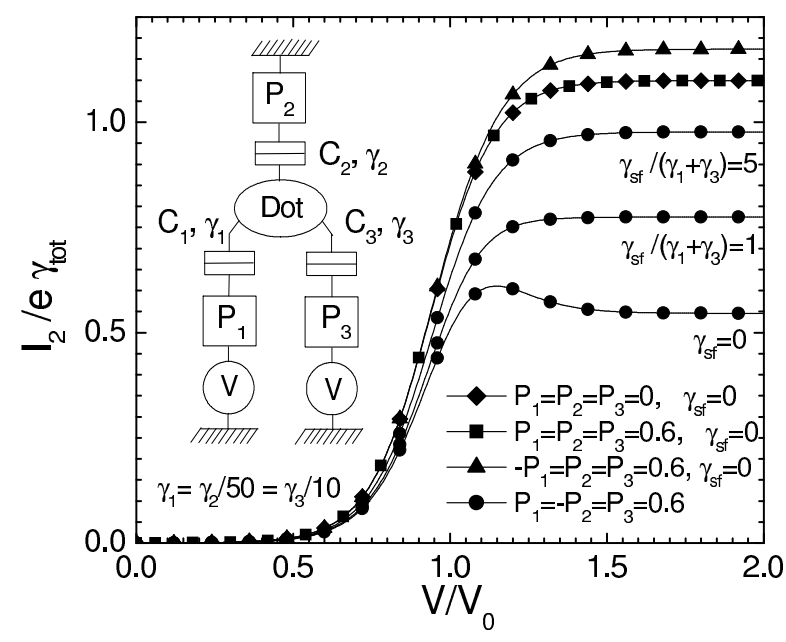

FIG. 1: Current-voltage characteristic of a quantum dot connected to three ferromagnetic leads $i \in\{1,2,3\}$, with respective polarizations $P_{i}$, through tunnel junctions with capacitances $C_{i}$ and net tunneling rates $\gamma_{i}$ (circuit shown in the inset). A voltage bias $V$ is applied to leads 1 and 3 ; lead 2 is connected to ground. The average current $I_{2}$ through lead 2 is shown as a function of voltage, for $C_{1}=C_{2}=C_{3}$, $\gamma_{1}=\gamma_{2} / 50=\gamma_{3} / 10, k_{B} T / E_{0}=0.1$, and different values of lead polarizations. The current is plotted in units of $e \gamma_{\text {tot }}=e \gamma_{2}\left(\gamma_{1}+\gamma_{3}\right) /\left(\gamma_{1}+\gamma_{2}+\gamma_{3}\right)$; the voltage in units of $V_{0}=E_{0} C /\left(C_{1}+C_{3}\right) e ; E_{0}$ is the position of the dot level. For $P_{1}=P_{2}=P_{3}, I_{2}$ coincides with the paramagnetic case (diamonds). In the other cases, the high-voltage limit of $I_{2}$ can be larger or smaller than the paramagnetic value, depending on the lead polarizations. For $P_{1}=-P_{2}=P_{3}=0.6$ (circles), the effect of spin-flip scattering is shown. Spin-flip scattering makes the $I_{2}-V$ curve tend to the paramagnetic one.

possibility of positive cross-correlations in the absence of correlated injection. Surprisingly, spin accumulation on the dot is not necessary to observe this effect. Furthermore, the sign of cross-correlations can be switched by reversing the magnetization of one contact. The effect is robust against spin-flips on the dot as long as the spin-flip scattering rate is less than the tunneling rates. 
The system we have in mind is a quantum dot connected to three ferromagnetic leads $i \in\{1,2,3\}$, through tunnel junctions with capacitances $C_{i}$ and net spinindependent tunneling rates $\gamma_{i}$ (inset of Fig. (1). A voltage bias $V$ is applied to leads 1 and 3; lead 2 is connected to ground. At voltages and temperatures much lower than the intrinsic level spacing and the charging energy $E_{C}=e^{2} / 2 C$ of the $\operatorname{dot}\left(C=\sum_{i} C_{i}\right)$, only one energy level of the dot located at $E_{0}$ needs to be taken into account. In this situation, the dot can either be empty, or occupied with one electron with spin $\sigma \in\{\uparrow, \downarrow\}$. Here and in the following, we will measure energies from the Fermi level, i.e., $E_{F}=0$.

The collinear magnetic polarizations $P_{j}$ of the leads are taken into account by using spin-dependent tunneling rates $\gamma_{j \sigma}=\gamma_{j}\left(1+\sigma P_{j}\right)$, where $\sigma= \pm 1$ labels the electron spin. In a simple model, the spin-dependence is a consequence of the different densities of states for majority and minority electrons [20]. The rate for an electron to tunnel on/off the $\operatorname{dot}(\epsilon= \pm 1)$ through junction $j$ is then given by $\Gamma_{j \sigma}^{\epsilon}=\gamma_{j \sigma} /\left(1+\exp \left[\epsilon\left(E_{0}-e V_{j}\right) / k_{B} T\right]\right)$, where $V_{1}=V_{3}=-C_{2} V / C, V_{2}=\left(C_{1}+C_{3}\right) V / C$. On the dot, there can be spin-flip scattering, for instance due to spin-orbit coupling or magnetic impurities. Here, we will assume that the on-site energy on the dot does not depend on spin. Hence, due to the detailed-balance rule, the spin-flip scattering rate $\gamma_{s f}$ does not depend on spin.

In the sequential-tunneling limit $\hbar \gamma_{j \sigma} \ll k_{B} T$, electronic transport through the dot can be described by the master equation [14, 19]:

$$
\frac{d}{d t}\left[\begin{array}{l}
p_{\uparrow}(t) \\
p_{\downarrow}(t) \\
p_{0}(t)
\end{array}\right]=\hat{M}\left[\begin{array}{l}
p_{\uparrow}(t) \\
p_{\downarrow}(t) \\
p_{0}(t)
\end{array}\right],
$$

where $p_{\psi}(t), \psi \in\{\uparrow, \downarrow, 0\}$, is the instantaneous occupation probability of state $\psi$ at time $t$, and where

$$
\hat{M}=\left[\begin{array}{ccc}
-\Gamma_{\uparrow}^{-}-\gamma_{s f} & \gamma_{s f} & \Gamma_{\uparrow}^{+} \\
\gamma_{s f} & -\Gamma_{\downarrow}^{-}-\gamma_{s f} & \Gamma_{\downarrow}^{+} \\
\Gamma_{\uparrow}^{-} & \Gamma_{\downarrow}^{-} & -\Gamma_{\uparrow}^{+}-\Gamma_{\downarrow}^{+}
\end{array}\right]
$$

depends on the total rates $\Gamma_{\sigma}^{\epsilon}=\sum_{j} \Gamma_{j \sigma}^{\epsilon}$ and $\gamma_{\sigma}=$ $\sum_{j} \gamma_{j \sigma}$. The stationary occupation probabilities $\bar{p}_{\psi}$ are

$$
\bar{p}_{\sigma}=\frac{\Gamma_{\sigma}^{+} \Gamma_{-\sigma}^{-}+\gamma_{s f}\left(\Gamma_{\sigma}^{+}+\Gamma_{-\sigma}^{+}\right)}{\gamma_{\sigma} \gamma_{-\sigma}-\Gamma_{\sigma}^{+} \Gamma_{-\sigma}^{+}+\gamma_{s f}\left(\Gamma_{\sigma}^{+}+\Gamma_{-\sigma}^{+}+\gamma_{\sigma}+\gamma_{-\sigma}\right)},
$$

and $\bar{p}_{0}=1-\bar{p}_{\uparrow}-\bar{p}_{\downarrow}$. They can be used to calculate the average value $\left\langle I_{j}\right\rangle$ of the tunneling current $I_{j}(t)$ through junction $j$ as $\left\langle I_{j}\right\rangle=e \sum_{\epsilon, \sigma} \epsilon \Gamma_{j \sigma}^{\epsilon} \bar{p}_{A(\sigma,-\epsilon)}$, where $A(\sigma, \epsilon)$ is the state of the dot after the tunneling of an electron with spin $\sigma$ in the direction $\epsilon$, i.e., $A(\sigma,-1)=0, A(\sigma,+1)=\sigma$.

In the following, we first consider the situation $E_{0}>0$. The voltage $V$ will always be assumed to be positive, such that it is energetically more favorable for electrons to go from the input electrode 2 to the output electrodes 1

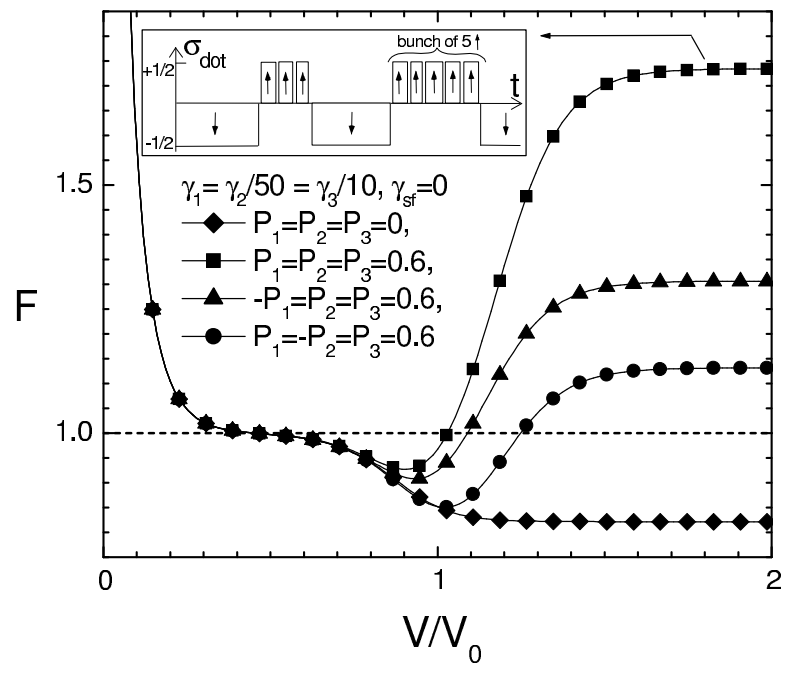

FIG. 2: Fano factor $F=S_{22} / 2 e I_{2}$ of lead 2 as a function of voltage, for the same circuit parameters as in Fig. 1. In all curves $\gamma_{s f}=0$. For $P_{1}=P_{2}=P_{3}$, the Fano factor is different from that of the paramagnetic case (diamonds) in contrast to what happens for the average currents. The inset shows the typical time dependence of the spin on the dot, in the high-voltage limit $V \gg V_{0}$ for the case $P_{1}=P_{2}=P_{3}=0.6$.

or 3 than in the opposite direction. The typical voltage dependence of $I_{2} \equiv\left\langle I_{2}\right\rangle$ is shown in Fig. 1] The total current $I_{2}$ is exponentially suppressed at low voltages, increases around a voltage $V_{0}=E_{0} C /\left(C_{1}+C_{3}\right) e$, and saturates at higher voltages. The width of the increase is determined by $k_{B} T / e$. The high-voltage limit of $I_{2}$ depends on the polarizations $P_{i}$ and rates $\gamma_{i}$ but not on the capacitances $C_{i}$. For a sample with magnetic contacts, this limit can be higher or lower than that of the paramagnetic case, depending on the parameters considered. In the high-voltage limit, $I_{2}\left(P_{1}, P_{2}, P_{3}\right)-I_{2}(0,0,0)=$ $2 e \gamma_{c} P_{\text {out }}\langle S\rangle$, where $P_{\text {out }}=\left(P_{1} \gamma_{1}+P_{3} \gamma_{3}\right) /\left(\gamma_{1}+\gamma_{3}\right)$ is the net output lead polarization, $\langle S\rangle=\nu\left(P_{2}-P_{\text {out }}\right)$ is the average spin accumulation on the $\operatorname{dot}$ [21] and $\gamma_{c}=\gamma_{2}\left(\gamma_{1}+\gamma_{3}\right) /\left(\gamma_{1}+2 \gamma_{2}+\gamma_{3}\right)$. Here, $\nu$ is a positive function of the polarizations, the tunneling and scattering rates, which tends to 0 at large $\gamma_{s f}$. Having a saturation current different from the paramagnetic case requires $P_{\text {out }} \neq 0$ and $\langle S\rangle \neq 0$. Spin-flip scattering modifies the $I_{2}-V$ curve once $\gamma_{s f}$ is of the order of the tunneling rates. It suppresses spin accumulation and makes the $I_{2}-V$ curve tend to the paramagnetic one.

The power spectrum of tunneling current correlations in leads $i$ and $j$ is defined as

$$
S_{i j}(\omega)=2 \int_{-\infty}^{+\infty} d t \exp (i \omega t)\left\langle\Delta I_{i}(t) \Delta I_{j}(0)\right\rangle
$$

where $\Delta I_{i}(t)=I_{i}(t)-\left\langle I_{i}\right\rangle$. The terms $\left\langle I_{i}(t) I_{j}(0)\right\rangle$ can be written as a function of the conditional probabilities $P_{\psi, \varphi}^{c}(t)$ which are the occupation probabilities of the state $\psi$ at time $t$ if at $t=0$ the state was $\varphi$, and which 
are zero for $t<0$. Solving Eq. (11) with the initial condition $P_{\psi, \varphi}^{c}(t=0)=\delta_{\psi, \varphi}$ leads to $P_{\psi, \varphi}^{c}(t)$. Its Fourier transform is given by $\hat{P}^{c}(\omega)=\int_{0}^{\infty} d t \exp (i \omega t) \hat{P}^{c}(t)=$ $-(i \omega+\hat{M})^{-1}$. The eigenvalues of the matrix $\hat{M}$ thus govern the frequency dependence of $\hat{P}^{c}(\omega)$. The nonzero eigenvalues are $\lambda_{ \pm}=\frac{1}{2}\left(-2 \gamma_{s f}-\gamma_{\uparrow}-\gamma_{\downarrow} \pm \Delta\right)$, with $\Delta^{2}=4 \gamma_{s f}^{2}+\left(\gamma_{\uparrow}-\gamma_{\downarrow}\right)^{2}-4 \gamma_{s f}\left(\Gamma_{\uparrow}^{+}+\Gamma_{\downarrow}^{+}\right)+4 \Gamma_{\uparrow}^{+} \Gamma_{\downarrow}^{+}$. This eventually leads to

$$
S_{i j}(\omega)=\delta_{i j} S_{j}^{S c h}+\sum_{\sigma, \sigma^{\prime}} S_{i, \sigma, j, \sigma^{\prime}}^{c}(\omega),
$$

where $S_{j}^{S c h}=2 e^{2} \sum_{\epsilon, \sigma} \Gamma_{j \sigma}^{\epsilon} \bar{p}_{A(-\epsilon, \sigma)}$ is the Schottky noise produced by tunneling through junction $j$, and

$$
\begin{aligned}
\frac{S_{i, \sigma, j, \sigma^{\prime}}^{c}(\omega)}{2 e^{2}} & =\sum_{\epsilon, \epsilon^{\prime}} \epsilon \epsilon^{\prime}\left[\Gamma_{i \sigma}^{\epsilon^{\prime}} G_{A\left(\sigma,-\epsilon^{\prime}\right), A\left(\sigma^{\prime}, \epsilon\right)}(\omega) \Gamma_{j \sigma^{\prime}}^{\epsilon} \bar{p}_{A\left(\sigma^{\prime},-\epsilon\right)}\right. \\
& \left.+\Gamma_{j \sigma^{\prime}}^{\epsilon^{\prime}} G_{A\left(\sigma^{\prime},-\epsilon^{\prime}\right), A(\sigma, \epsilon)}(-\omega) \Gamma_{i \sigma}^{\epsilon} \bar{p}_{A(\sigma,-\epsilon)}\right] .
\end{aligned}
$$

Here, we defined $G_{\psi, \varphi}(\omega)=P_{\psi, \varphi}^{c}(\omega)+\bar{p}_{\psi} / i \omega$.

Equation (5) determines the full frequency-dependent tunneling current correlation functions of the threeterminal quantum dot. For frequencies larger than the cutoff frequencies $\lambda_{-}$and $\lambda_{+}$, the spectrum $S_{i j}(\omega)$ tends to the uncorrelated spectrum $\delta_{i j} S_{j}^{S c h}$. In the following, we will consider mainly the zero-frequency limit of $S_{i j}(\omega)$, because the frequencies $\lambda_{ \pm} \sim \gamma_{i}$ are difficult to access in experiment. Note that at zero frequency, the contribution of the screening currents ensuring electroneutrality of the capacitors after a tunneling event [8] is zero, i.e., $S_{i j} \equiv S_{i j}(0)$ is the signal measured in practice 22 .

Figures 2] 3] show the Fano factor $F=S_{22} / 2 e I_{2}$ and the cross-correlations $S_{13}$ as a function of $V$ for $\gamma_{s f}=0$. Well below $V_{0}$ the current is due to thermally activated tunneling and the noise is Poissonian. At very low voltage, $e V \leq k_{B} T$, the cross-over to thermal noise is observed. Around $V=V_{0}, F$ and $S_{13}$ show a step or a dip. The high-voltage limit strongly depends on tunneling rates and polarizations. In the paramagnetic case, the limit of $F$ lies in the interval $[1 / 2,1]$, and that of $S_{13} / 2 e I_{2}$ in $[-1 / 8,0]$. In the ferromagnetic case the highvoltage limit of $F$ can be either sub- or super-Poissonian, as already pointed out in the two-terminal case 18. Spin accumulation is not a necessary condition for having a super-Poissonian Fano factor, as can be seen for $P_{1}=P_{2}=P_{3}$, where $\langle S\rangle=0$. In this case, the essential point is that the current can flow only in short time windows where the current transport is not blocked by a down spin, see the inset of Fig. 2 This dynamical spin blockade leads to a bunching of tunneling events, and explains the super-Poissonian Fano factor.

The cross-correlations can be either positive or negative, see Fig. 3. Note that a super-Poissonian $F$ does not

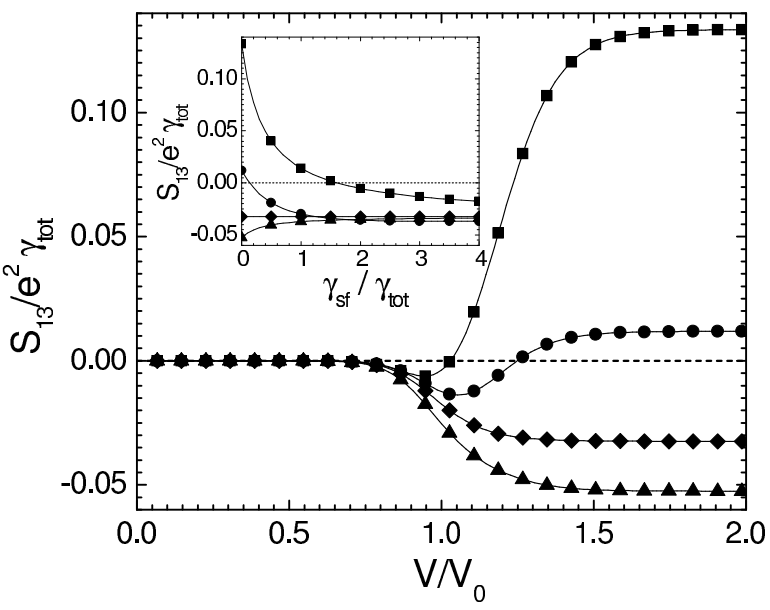

FIG. 3: Current cross-correlations between leads 1 and 3 as a function of voltage. The curves are shown for the same circuit parameters as in Fig. 2 The cross-correlations can be positive in the cases $P_{1}=-P_{2}=P_{3}=0.6$ (circles) and $P_{1}=P_{2}=P_{3}=0.6$ (squares). Note that the sign of crosscorrelations can be reversed just changing the sign of $P_{1}$. In all curves $\gamma_{s f}=0$. The inset shows the influence of spinflip scattering on the cross-correlations in the high-voltage limit $V \gg V_{0}$. In the paramagnetic case (diamonds), spin-flip scattering has no effect. In the limit $\gamma_{s f} \gg \gamma_{t o t}$, the crosscorrelations tend to the paramagnetic value.

necessarily imply positive cross-correlations, as shown by the case $-P_{1}=P_{2}=P_{3}=0.6$ in Figs. 2 [3] for which the cross-correlations are even more negative than in the paramagnetic case. Indeed, relations (5) and (6) together with charge conservation imply that $S_{22}-S_{2}^{S c h}=$ $\sum_{\sigma, \sigma^{\prime}} S_{1, \sigma, 3, \sigma^{\prime}}^{c}\left(\gamma_{1 \sigma}+\gamma_{3 \sigma}\right)\left(\gamma_{1 \sigma^{\prime}}+\gamma_{3 \sigma^{\prime}}\right) / \gamma_{1 \sigma} \gamma_{3 \sigma^{\prime}}$ at $V \gg$ $V_{0}$. Thus, at $V \gg V_{0}$, a super-Poissonian $F$ is equivalent to positive cross-correlations only at large voltages and if the two output leads have identical polarisations. For the case $-P_{1}=P_{2}=P_{3}=0.6$, cross-correlations are negative in spite of the super-Poissonian $F$ because the correlated electrons are mostly up electrons flowing through lead 3 . We note here that $\operatorname{Re}\left[S_{13}(\omega)\right]$ can change sign for intermediate frequencies and vanishes for $\omega \gg \lambda_{+}, \lambda_{-}$ [11.

We now briefly comment on the case $E_{0}<0$. For $V \gg V_{0}^{\prime}=-E_{0} C / C_{2} e$, the values of $I_{2}, F$ and $S_{13}$ are the same as previously. When $V$ is smaller than $V_{0}^{\prime}$, the most striking difference is that $F$ is polarizationdependent and thus not necessarily Poissonian.

The effect of spin-flip scattering is shown in the inset of Fig. 3. Spin-flip scattering influences the crosscorrelations once $\gamma_{s f}$ is of the order of the tunneling rates. In the high- $\gamma_{s f}$ limit, cross-correlations tend to the paramagnetic case for any value of the polarizations. Thus, strong elastic spin-flip scattering suppresses positive cross-correlations, in contrast to what happens with inelastic scattering in 12. In practice, experiments with a quantum dot connected to ferromagnetic leads and 


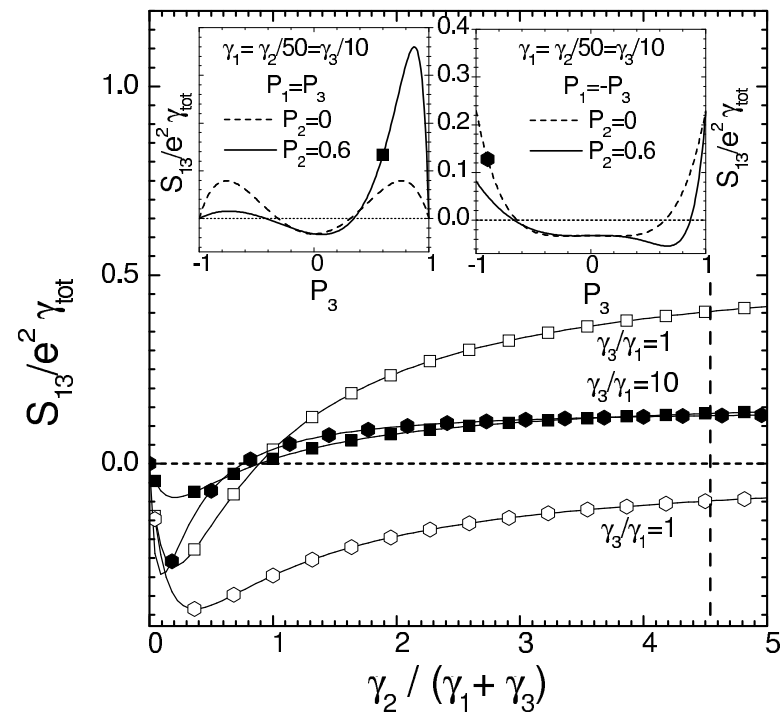

FIG. 4: Influence of asymmetry between $\gamma_{2}$ and $\gamma_{1}+\gamma_{3}$ on the high-voltage limit of the cross-correlations, for $P_{1}=P_{3}=$ $P_{3}=0.6$ (squares) and $-P_{1}=P_{3}=0.9, P_{2}=0$ (hexagons), for $\gamma_{3} / \gamma_{1}=10$ (full symbols) and $\gamma_{3} / \gamma_{1}=1$ (empty symbols). Large values of $\gamma_{2} /\left(\gamma_{1}+\gamma_{3}\right)$ favor positive cross-correlations. For $-P_{1}=P_{3}=0.9, P_{2}=0$, an asymmetry between $\gamma_{1}$ and $\gamma_{3}$ is also necessary. The vertical dashed line indicates the ratio $\gamma_{2} /\left(\gamma_{1}+\gamma_{3}\right)$ corresponding to Figs. 12 The two insets show the high-voltage limit of the cross-correlations as a function of $P_{3}$, for $\gamma_{1}=\gamma_{2} / 50=\gamma_{3} / 10, P_{1}=P_{3}$ (left inset) and $P_{1}=-P_{3}$ (right inset) and $P_{2}=0$ (dashed lines) or $P_{2}=0.6$ (full lines). For all curves $\gamma_{s f}=0$.

$\gamma_{s f} \ll \gamma_{t o t}$ have already been performed [23]. Thus, spin-flip scattering should not prevent the observation of positive cross-correlations in quantum dots.

Finally, we address the problem of how to choose parameters that favor the observation of positive crosscorrelations. First, finite lead polarizations are necessary 17], see the insets of Fig. [4. However, it is possible to get positive cross-correlations even if $P_{2}=0$, provided the output leads 1,3 of the device are sufficiently polarized (dashed lines in the insets of Fig. (4). The case where the three electrodes are polarized in the same direction seems the most favorable. In the high-voltage limit, choosing $P_{1}=P_{2}=P_{3}$ and $\gamma_{s f}=0$ leads to

$$
S_{13}=\frac{16 e^{2} \gamma_{1} \gamma_{2}^{2} \gamma_{3}\left[\left(\gamma_{1}+2 \gamma_{2}+\gamma_{3}\right) P_{1}^{2}-\gamma_{1}-\gamma_{3}\right]}{\left(\gamma_{1}+\gamma_{3}\right)\left(\gamma_{1}+2 \gamma_{2}+\gamma_{3}\right)^{3}\left(1-P_{1}^{2}\right)} .
$$

The asymmetry between the tunneling rates $\gamma_{i}$ has a

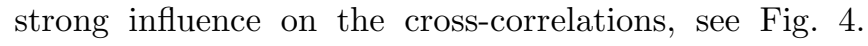
Large values of $\gamma_{2} /\left(\gamma_{1}+\gamma_{3}\right)$ favor the observation of positive cross-correlations, see e. g. Eq. (7), by decreasing $\bar{p}_{0}$. This allows to extend the domains of positive cross-correlations to smaller values of the polarizations, which is important because experimental contact materials are not fully polarized. For $\gamma_{1}=\gamma_{2} / 10=\gamma_{3}$, the polarizations $P_{1}=P_{2}=P_{3}=0.4$ typical for Co
24] lead to positive cross-correlations of the order of $S_{13} / e^{2} \gamma_{t o t} \simeq 0.08$. With $\gamma_{t o t} \simeq 5 \mathrm{GHz}$ this corresponds to $10^{-29} \mathrm{~A}^{2} \mathrm{~s}$, a noise level accessible with present noiseamplification techniques [16].

In conclusion, we have demonstrated that transport through a multi-terminal quantum dot with ferromagnetic contacts is characterized by a new mechanism, viz., dynamical spin blockade. As one of its consequences we predict positive current cross-correlations in the drain contacts without requiring the injection of correlated electron pairs. We have included spin-flip scattering on the dot and have shown that the effect persists as long as the spin-flip scattering rate is less than the tunneling rate to the contacts.

We thank T. Kontos and C. Schönenberger for useful discussions. This work was financially supported by the RTN Spintronics, by the Swiss NSF, and the NCCR Nanoscience.

[1] Ya. M. Blanter and M. Büttiker, Phys. Rep. 336, 1 (2000).

[2] Quantum Noise in Mesoscopic Physics, edited by Yu. V. Nazarov (Kluwer, Dordrecht, 2003).

[3] V. A. Khlus, Sov. Phys. JETP 66, 1243 (1987).

[4] G. B. Lesovik, JETP Lett. 49, 592 (1989).

[5] M. Büttiker, Phys. Rev. Lett. 65, 2901 (1990).

[6] M. Büttiker, Phys. Rev. B 46, 12485 (1992).

[7] M. Henny et al., Science 284, 296 (1999); W. D. Oliver et al., Science 284, 299 (1999); S. Oberholzer et al., Physica (Amsterdam) E 6, 314 (2000).

[8] See the article of M. Büttiker, in Ref. [2].

[9] T. Martin, Phys. Lett. A 220, 137 (1996); M. P. Anantram and S. Datta, Phys. Rev. B 53, 16390 (1996); J. Torres and T. Martin, Eur. Phys. J. B 12, 319 (1999); T. Gramespacher and M. Büttiker, Phys. Rev. B 61, 8125 (2000); J. Torres et al., ibid. 63, 134517 (2001); J. Börlin et al., Phys. Rev. Lett. 88, 197001 (2002); P. Samuelsson and M. Büttiker, ibid. 89, 046601 (2002); Phys. Rev. B 66, 201306 (2002); F. Taddei and R. Fazio, ibid. 65, 134522 (2002).

[10] A. M. Martin and M. Büttiker, Phys. Rev. Lett. 84, 3386 (2000).

[11] A. Cottet et al., to be published elsewhere.

[12] C. Texier and M. Büttiker, Phys. Rev. B 62, 7454 (2000).

[13] I. Safi et al., Phys. Rev. Lett. 86, 4628 (2001); A. Crepieux et al., Phys. Rev. B 67, 205408 (2003).

[14] A. N. Korotkov, Phys. Rev. B 49, 10381 (1994); S. Hershfield et al., ibid. 47, 1967 (1993); U. Hanke et al., ibid. 48, 17209 (1993).

[15] D. Loss and E. V. Sukhorukhov, Phys. Rev. Lett. 84, 1035 (2000); E. V. Sukhorukov et al., Phys. Rev. B 63, 125315 (2001); D.V. Averin, in Macroscopic Quantum Coherence and Quantum Computing, edited by D.V. Averin, B. Ruggiero, and P. Silvestrini (Kluwer, Dordrecht, 2001); cond-mat/0010052

[16] H. Birk et al., Phys. Rev. Lett. 75, 1610 (1995).

[17] D. A. Bagrets and Yu. V. Nazarov, Phys. Rev. B 67, 085316 (2003). 
[18] B. R. Bulka et al., Phys. Rev. B 60, 12246 (1999).

[19] B. R. Bulka, Phys. Rev. B 62, 1186 (2000).

[20] M. Julliere, Phys. Lett. A 54, 225 (1975).

[21] J. Barnas and A. Fert, Phys. Rev. Lett. 80, 1058 (1998); S. Takahashi and S. Maekawa, ibid. 80, 1758 (1998); J. Barnaś and A. Fert, Europhys. Lett. 44, 85 (1998); F. Guinea, Phys. Rev. B 58, 9212 (1998); H. Imamura et al., ibid. 59, 6017 (1999); A. Brataas et al., Eur. Phys. J. B 9, 421 (1999); X.H. Wang and A. Brataas, Phys. Rev.
Lett. 83, 5138 (1999).

[22] The total current correlations, including screening currents, are $S_{i j}^{\text {tot }}(\omega)=$ $\sum_{n, m}\left(\delta_{i, n}-\frac{C_{i}}{C}\right)\left(\delta_{j, m}-\frac{C_{j}}{C}\right) S_{n m}(\omega)$.

[23] M. Deshmukh and D. C. Ralph, Phys. Rev. Lett. 89, 266803 (2002).

[24] M. Soulen et al., Science 282, 85 (1998). 